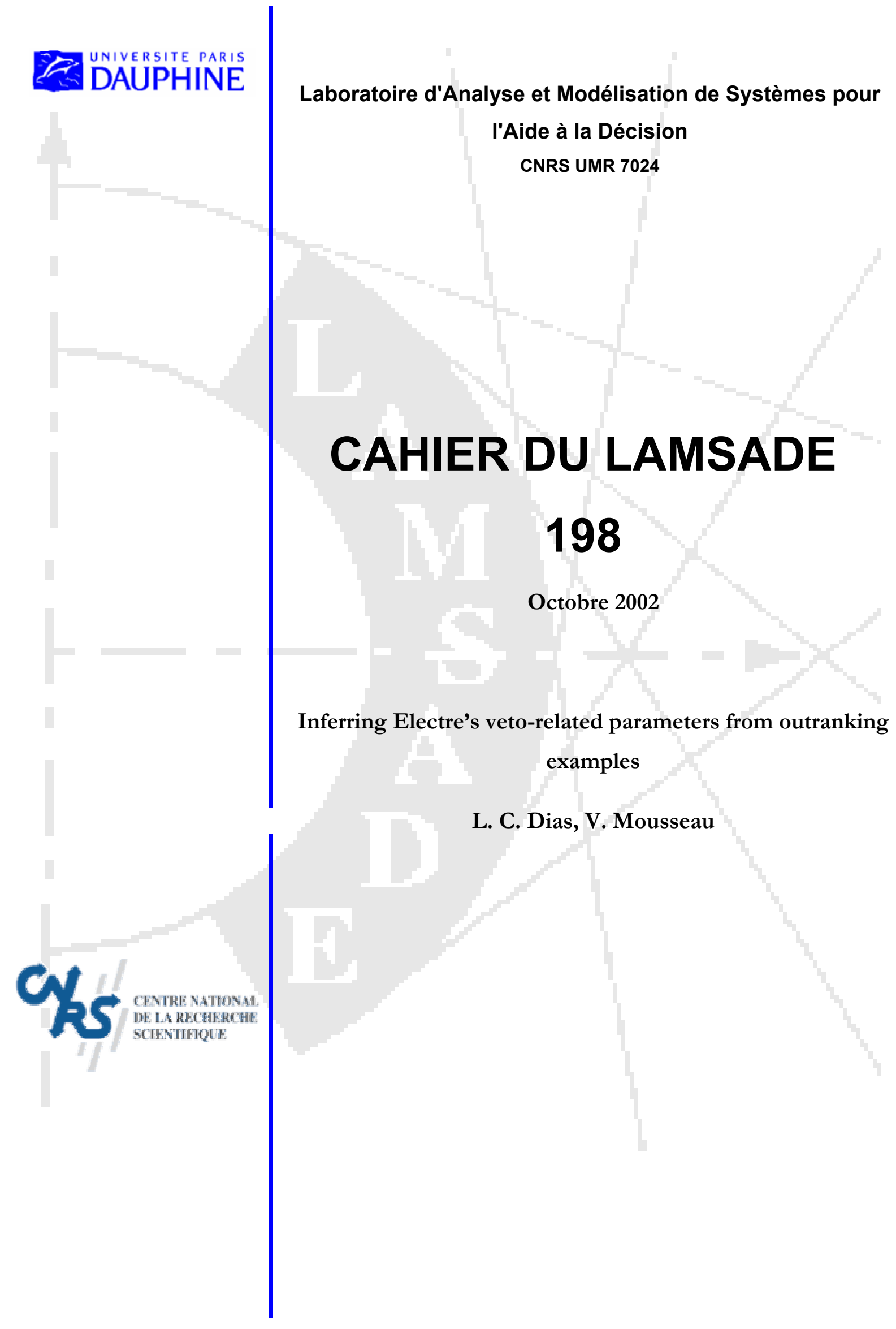




\title{
Inferring Electre's veto-related parameters from outranking examples
}

\author{
Luis C. Dias* ${ }^{*}$ and Vincent Mousseau ${ }^{\ddagger}$
}

3 septembre 2002

${ }^{*}$ Faculty of Economics, University of Coimbra, Av. Dias da Silva, 3004-512 Coimbra, Portugal, tel: (351) 239-790500

${ }^{\dagger}$ INESC Coimbra, R. Antero de Quental 199, 3000-033 Coimbra, Portugal, e-mail: Idias@pombo.inescc.pt ${ }^{\ddagger}$ DIMACS, Rutgers University, CoRE Building, 96 Frelinghuysen Road Piscataway NJ 08854-8018 USA

${ }^{\S}$ LAMSADE, Université Paris Dauphine, Place du Maréchal De Lattre de Tassigny, 75775 Paris Cedex 16, France, tel: (33-1)44-05-44-01, fax: (33-1)44-05-40-91, e-mail: mousseau@lamsade.dauphine.fr 


\section{Table des matières}

Table of Contents $\quad$ ii

Résumé

$\begin{array}{ll}\text { Abstract } & \text { iv }\end{array}$

$\begin{array}{ll}\text { Introduction } & 1\end{array}$

1 Valued Outranking Relations in Electre $\quad 2$

1.1 Outranking relations for a single criterion . . . . . . . . . . . . . 2

1.2 Concordance relation . . . . . . . . . . . . . . . . 2

1.3 Non-discordance relations . . . . . . . . . . . . . . . 3

1.4 Valued outranking relations . . . . . . . . . . . . . . . 4

2 Inference of parameter values from crisp outranking statements 5

3 Inference of veto-related parameters for a single criterion 6

3.1 Inference of $v_{i}$ considering $S(a, b) \ldots \ldots \ldots \ldots \ldots$

3.2 Inference of $u_{i}$ and $v_{i}$ considering $S^{\prime}(a, b) \ldots \ldots \ldots \ldots$

3.3 Inference of $u_{i}$ and $v_{i}$ considering $S^{\prime \prime}(a, b) \ldots \ldots \ldots$

4 Inference of all veto-related parameters simultaneously 9

4.1 Inference of $v_{i}$ considering $S(a, b)$ or $S^{\prime}(a, b) \ldots \ldots \ldots \ldots$

4.2 Inference of $u_{i}$ and $v_{i}$ considering $S^{\prime \prime}(a, b) \ldots \ldots \ldots \ldots$

5 Illustrative example $\quad 13$

5.1 Scheme of the experiment . . . . . . . . . . . . . . 13

5.2 inferring one veto using the relation $S \ldots \ldots \ldots \ldots \ldots$

5.3 inferring one veto using the relation $S^{\prime} \ldots \ldots \ldots \ldots$

5.4 inferring one veto using the relation $S^{\prime \prime} \ldots \ldots \ldots \ldots \ldots$

5.5 inferring several veto using the relation $S^{\prime \prime} \ldots \ldots \ldots \ldots$

$\begin{array}{lr}\text { Conclusion } & 16\end{array}$

$\begin{array}{ll}\text { Acknowledgements } & 17\end{array}$

$\begin{array}{ll}\text { References } & 17\end{array}$ 


\section{Inférence des seuils de veto d'Electre à partir d'exemples de surclassement}

\section{Résumé}

Lorsque l'on considère les relations de surclassement valuées d'Electre, il est difficile d'intégrer la discordance (effets de veto) aux méthodologies d'agrégationdésagrégation. Nous présentons une procédure d'inférence partielle pour calculer la valeur des seuils de veto qui restitue au mieux des affirmations de surclassement fournies par un décideur (i.e., des exemples que le modèle Electre doit restituer).

Cet article poursuit des travaux antérieurs sur l'inference des autres paramètres préférentiels (coefficients d'importance, niveau de coupe, limites des catégories, ...) en direction d'une approche intégrée de l'inférence dans les méthodes Electre. Nous proposons des programmes mathématiques pour inférer les paramètres liés au veto, tout d'abord dans le cas d'un seul veto, puis dans le cas de veto sur plusieurs critères, et ceci en utilisant la relation de surclassement floue d'Electre III et deux variantes.

Ces programmes d'inférence partielle sont considérés comme des routines à utiliser de façon répétitive dans un processus de désagrégation interactif dans lequel le décideur révise l'information qu'il fournit à mesure qu'il obtient des résultats et apprend à propos de ses préférences.

Mots-Clés: Relations de Surclassement floues, Effet de veto, Inférence des paramètres, Electre 


\title{
Inferring Electre's veto-related parameters from outranking examples
}

\begin{abstract}
When considering Electre's valued outranking relations, aggregation/disaggregation methodologies have difficulties in taking discordance (veto) into account. We present a partial inference procedure to compute the value of the veto-related parameters that best restore a set of outranking statements, provided by a decision maker (i.e., examples that an Electre model should restore).

This paper complements previous work on the inference of other preference-related parameters (weights, cutting level, category limits, ...), advancing toward an integrated approach to inference problems in Electre III and Tri methods. We propose mathematical programs to infer veto-related parameters, first considering only one criterion, then all criteria simultaneously, using the original version of Electre outranking relation and two variants. Depending on the case, these inference procedures lead to linear programming, 0-1 linear programming, or separable programming problems.
\end{abstract}

The partial inference programs are considered as problems to be solved several times in an interactive process, where decision makers continuously revise the information they provide as they learn from the results.

Keywords: Parameter Inference Procedures, Valued Outranking Relations, Electre, discordance, veto 


\section{Introduction}

The use of multiple criteria evaluation methods is often hindered by the need to provide precise values for many preference-related parameters whose role is not clear to the Decision Maker (DM). Aggregation/disaggregation methodologies, which have received much attention lately (see EJOR 30(2), [4]), allow to infer values for such parameters from holistic judgments (i.e., model results) that the DM is able to provide. Usually, when the underlying evaluation model is of the Electre type (cf. [5], [7]) or of the value function type (cf. [3], [16]), a mathematical programming problem is solved to find the combination of parameter values that best restores the examples of results proposed by the DM, by minimizing some error function. This is particularly useful if the inference procedure is part of an interactive process, where the DM observes whether his/her result examples can be restored, and reacts accordingly. If the examples indicated by the DM can be restored, he/she may explore the complete set of results corresponding to the multiple combinations of parameter values that satisfy the imposed conditions (robustness analysis), which may help him/her provide further information. If not, the DM has to discover which of those examples are inconsistent, in order to withdraw some of them (inconsistency analysis).

[2] have proposed this type of methodology, integrating parameters inference, robustness analysis, and inconsistency analysis for decision aiding based on the Electre Tri method (for an overview of Electre methods see [11] and [12]). However, the implementation they proposed was limited to the situations where Electre's veto thresholds were not taken into account.

Inferring all the parameters in Electre simultaneously requires solving non-linear programs with non-convex constraints (see [7]), which are usually difficult. The current paper follows a different strategy, based on inferring a subset of the parameter values at a time, while maintaining the remaining ones fixed. These "partial" inference problems, besides simplifying the mathematical programs to be solved, present important advantages. First, they let the DM focus his/her attention on a subset of parameters at a time (e.g., concordance-related parameters, then discordance-related parameters, then returning to concordance, etc.). Second, they allow the DM to control the interactive process in an easier way. Namely, when there are many alternative combinations of parameter values that satisfy all the requests of the DM, keeping a subset of the parameters temporarily constant prevents the solutions from being too disparate (even among alternative optima). Furthermore, the DM is less likely to encounter radically different solutions when progressing from one iteration to the next one, and is able to better understand the consequences of changing the examples. Indeed, we believe that inference programs should not be considered as a problem to be solved only once, but rather as problems to be solved several times in an interactive learning process, where the DM continuously revises the information he/she provides as he/she learns from the results of the inference programs.

While [6] considers that the concordance-related parameters were the only variables, this paper now considers that all parameters are fixed except the discordance-related ones (Electre's veto thresholds). Both papers apply to the Electre methods that use valued outranking relations (Electre III and Tri), although this work has been motivated by the application to the Electre Tri method. The holistic information provided by the DM consists of pairs of alternatives $(a, b)$ such that, to his/her opinion, " $a$ outranks $b$ " (denoted $a S b$ ), or " $a$ does not outrank 
$b$ " (denoted $\neg a S b$ ). Examples of results from the Electre Tri method may easily be translated into outranking statements of this kind. This paper intends to present how to infer Electre's veto thresholds from a set of such "crisp" outranking statements.

The following section presents the original outranking relation, as well as two variants we proposed in [6]. Section 2 presents the inference problem in a general format. Section 3 considers the problem of inferring the veto parameters for one criterion at a time, as a simplification of the more general problem that may nevertheless be useful in practice, since the veto parameters are not inter-related among different criteria. Section 4 deals with the more general problem of inferring the veto parameters for more than one criterion simultaneously. Finally, Section 5 presents illustrative examples, and a closing section offers a summary and some conclusions.

\section{Valued Outranking Relations in Electre}

In this section we recall how Electre III (see [10]) and Electre Tri (see [15], [12]) build a valued outranking relation on the set of alternatives. Let $A$ denote a finite set of alternatives characterized by their evaluations on $n$ criteria $g_{1}, \ldots, g_{n} . g_{j}(a)$ denotes the evaluation of an alternative $a \in A$ on criterion $g_{j}$. Without any loss of generality, we will assume that the evaluations are coded in such a way that the higher the value, the better it is.

\subsection{Outranking relations for a single criterion}

Electre builds, for each criterion $g_{j}$, a valued outranking relation $S_{j}$ restricted to a single criterion. For any ordered pair $(a, b) \in A^{2}, S_{j}(a, b)$ is defined by (1) on the basis of $g_{j}(a), g_{j}(b)$ and two thresholds: indifference $q_{j}$ and preference $p_{j}\left(0 \leq q_{j}<p_{j}{ }^{1}\right) . S_{j}(a, b)$ represents the degree to which alternative $a$ outranks (is at least as good as) $b$. In this paper, we consider the thresholds $p_{j}$ and $q_{j}$ as constant, although it is possible to consider them as affine functions (see [1]).

$$
S_{j}(a, b)=\left\{\begin{array}{l}
0, \quad \text { if } g_{j}(b)-g_{j}(a) \geq p_{j} \\
\frac{p_{j}-g_{j}(b)+g_{j}(a)}{p_{j}-q_{j}}, \quad \text { if } q_{j}<g_{j}(b)-g_{j}(a)<p_{j} \\
1, \quad \text { if } g_{j}(b)-g_{j}(a) \leq q_{j}
\end{array}\right.
$$

\subsection{Concordance relation}

The valued concordance relation $C(a, b)$ is grounded on the relations $S_{j}(j=1,2, \ldots, n)$ and represents the level of majority among the criteria in favor of the assertion " $a$ is at least as good as $b$ ". When computing this majority level, each criterion $g_{j}$ has a weight $w_{j} \geq 0$ representing its voting power. Without any loss of generality, we will consider $\sum_{j=1}^{n} w_{j}=1$. Therefore, $C(a, b)$ can be written as follows:

$$
C(a, b)=\frac{1}{\sum_{j=1}^{n} w_{j}} \sum_{j=1}^{n} w_{j} . S_{j}(a, b)=\sum_{j=1}^{n} w_{j} . S_{j}(a, b)
$$

1. We will consider $q_{j}<p_{j}$, although Electre also allows $q_{j}=p_{j}$. 


\subsection{Non-discordance relations}

Electre builds, for each criterion $g_{j}$, a valued discordance relation $d_{j}$ restricted to that criterion. This relation $d_{j}(a, b)$ is defined by (3) on the basis of $g_{j}(a), g_{j}(b)$, a veto threshold $v_{j}$ and a preference threshold $p_{j}\left(p_{j}<v_{j}{ }^{2}\right)$ (see Figure 1). In this paper, we consider the thresholds $v_{j}$ (as we already did for $p_{j}$ and $q_{j}$ ) as constant, although it is possible to consider them as affine functions (see [1]).

$$
d_{j}(a, b)=\left\{\begin{array}{l}
1, \quad \text { if } g_{j}(b)-g_{j}(a) \geq v_{j} \\
\frac{g_{j}(b)-g_{j}(a)-p_{j}}{v_{j}-p_{j}}, \quad \text { if } p_{j}<g_{j}(b)-g_{j}(a)<v_{j} \\
0, \quad \text { if } g_{j}(b)-g_{j}(a) \leq p_{j}
\end{array}\right.
$$

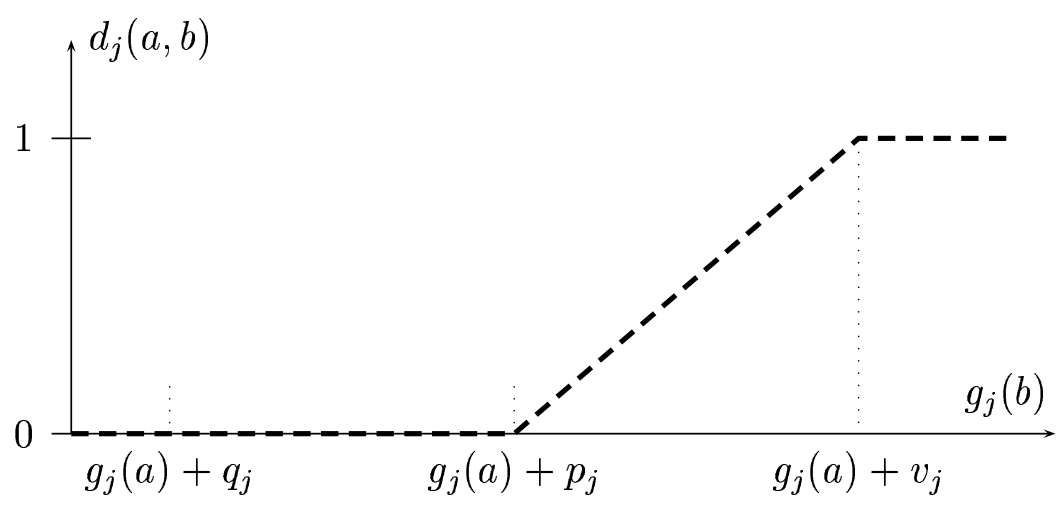

FIG. 1: Partial valued outranking relation

An overall valued non-discordance relation $N D(a, b)$ is grounded on $C(a, b)$ and on the relations $d_{j}, j=1,2, \ldots, n$; it represents the degree to which the minority criteria collectively oppose a veto to the assertion " $a$ is at least as good as $b$ ". A classical way of defining $N D(a, b)$ is given in (4). $N D(a, b)=0$ corresponds to a situation where the minority criteria are totally opposed to $a S b$ whereas $N D(a, b)=1$ means that none of the criteria oppose a veto to $a S b$.

$$
N D(a, b)=\prod_{j \in \bar{F}} \frac{1-d_{j}(a, b)}{1-C(a, b)} \text { where } \bar{F}=\left\{j \in F / d_{j}(a, b)>C(a, b)\right\}
$$

This expression is equivalent to (5):

$$
N D(a, b)=\prod_{j \in F} N D_{j}(a, b)
$$

where $^{3}$ :

$$
N D_{j}(a, b)=\operatorname{Min}\left\{1, \frac{1-d_{j}(a, b)}{1-C(a, b)}\right\} .
$$

2. We will consider $p_{j}<v_{j}$, although Electre also allows $p_{j}=v_{j}$.

3 . Let us remark that we can state $C(a, b)<1$, as the case $C(a, b)=1$ corresponds to a situation where no discordant criterion exists. 
As an alternative, [6] propose the valued non-discordance relation defined by (7)-(8), where $u_{j} \in\left[p_{j}, v_{j}\right.$ is a new parameter for the $j$-th criterion:

$$
\begin{gathered}
N D^{\prime}(a, b)=\prod_{j \in F} N D_{j}^{\prime}(a, b)=\prod_{j \in F}\left(1-d_{j}^{\prime}(a, b)\right) \\
d_{j}^{\prime}(a, b)=\left\{\begin{array}{l}
1 \text { if } g_{j}(b)-g_{j}(a) \geq v_{j} \\
\frac{g_{j}(b)-g_{j}(a)-u_{j}}{v_{j}-u_{j}} \\
0 \text { if } g_{j}(b)-g_{j}(a) \leq g_{j}(b)-g_{j}(a)<v_{j}
\end{array}\right.
\end{gathered}
$$

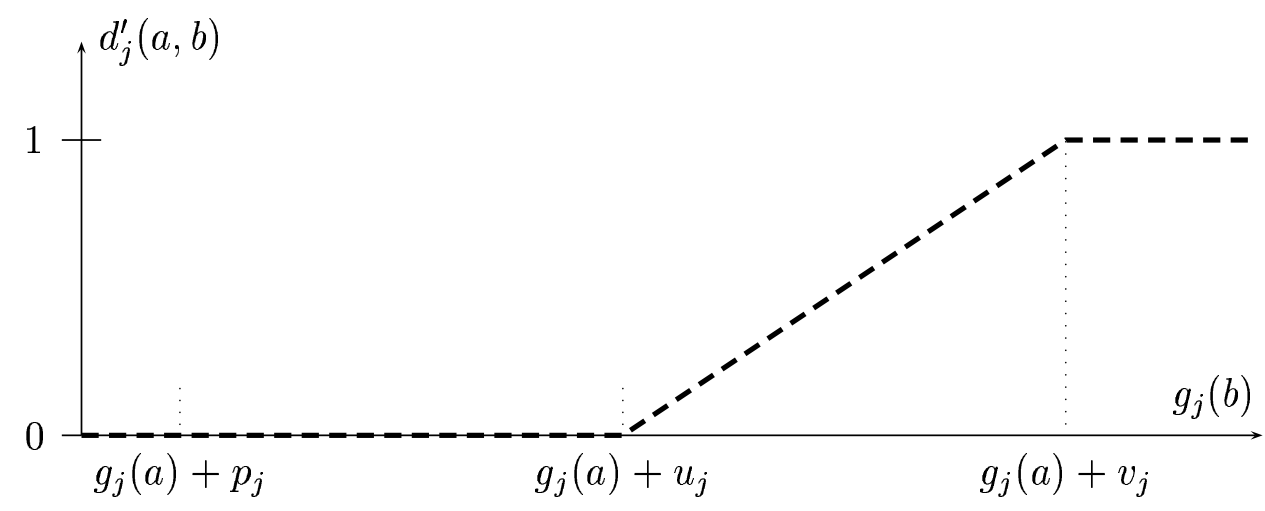

FIG. 2: Partial discordance relation $d_{j}^{\prime}(a, b)$

The new threshold $u_{j}$ defines the difference of performances in favor of $b$ where the discordance starts weakening the outranking relation (see Figure 2). It can be considered either:

- as an additional preference parameter to be elicited directly through an interaction with the DM, or indirectly using a disaggregation procedure, or

- as a technical parameter (rather than a preference-related one) that defines the extent to which differences of evaluation $g_{j}(b)-g_{j}(a)<v_{j}$ should (or should not) weaken the concordance $C(a, b)$ in the definition of $S(a, b)$ (a reasonable value for $u_{j}$ is $p_{j}+0.75\left(v_{j}-\right.$ $\left.p_{j}\right)$, as discussed in [6]).

A second alternative proposed by [6] to define a valued non-discordance relation is the following:

$$
N D^{\prime \prime}(a, b)=M i n_{j \in F} N D_{j}^{\prime}(a, b)
$$

\subsection{Valued outranking relations}

Electre combines the concordance and non-discordance relations in order to define the outranking relation $S$ as shown in (10)

$$
S(a, b)=C(a, b) . N D(a, b),
$$


or, according to the two alternative definitions,

$$
\begin{aligned}
& S^{\prime}(a, b)=C(a, b) \cdot N D^{\prime}(a, b) \\
& S^{\prime \prime}(a, b)=C(a, b) \cdot N D^{\prime \prime}(a, b)
\end{aligned}
$$

From the valued outranking relation $S(a, b)$, it is possible to define a family of nested crisp outranking relations $S_{\lambda}$; these crisp relations correspond to $\lambda$-cuts of $S(a, b)$, where the cutting level $\lambda \in[0.5,1]$ represents the minimum value for $S(a, b)$ so that $a S_{\lambda} b$ holds. The same applies when we consider $S^{\prime}(a, b)$ or $S^{\prime \prime}(a, b)$ instead of $S(a, b)$.

\section{Inference of parameter values from crisp outranking sta- tements}

The construction of the relation $S$ (or $S^{\prime}$, or $S^{\prime \prime}$ ) involves determining the evaluation vector of the alternatives, and setting many parameters: the criteria weights, the various thresholds, and the cutting level. DMs often find it difficult to provide precise values for all these preference parameters. Hence, "disaggregation approaches" have been proposed to infer the parameter values from holistic judgments.

Let us consider a decision process in which the DM is not able (or not willing) to assign directly values to the preference parameters involved in an outranking relation, but can state crisp statements about this relation for some specific pairs of alternatives $(a, b)$, either positive $(a S b)$ or negative $(\neg a S b)$. Let us denote $S^{+}=\left\{(a, b) \in A^{2}\right.$ such that the DM stated $\left.a S b\right\}$ and $S^{-}=\left\{(a, b) \in A^{2}\right.$ such that the DM stated $\left.\neg a S b\right\}$. Then, a combination of parameter values is able to restore the DM's request iff $S(a, b) \geq \lambda, \forall(a, b) \in S^{+}$and $S(a, b)<\lambda, \forall(a, b) \in S^{-}$. The system of constraints below (13) has a solution if and only if there exists a combination of parameter values that yields all the crisp outranking statements in $S^{+}$and $S^{-}$. Some additional constraints can be added to this system, in order to integrate explicit statements of the DM concerning the values of some parameters.

$$
\left\{\begin{array}{l}
S(a, b) \geq \lambda, \quad \forall(a, b) \in S^{+} \\
S(a, b)<\lambda, \quad \forall(a, b) \in S^{-} \\
\lambda \in[0.5,1] \\
v_{j}>p_{j}>q_{j} \geq 0, \quad \forall j \in F \\
\sum_{j=1}^{n} w_{j}=1 ; \quad w_{j} \geq 0, \forall j \in F
\end{array}\right.
$$

The idea of inferring all the parameters by maximizing the minimum slack for the above system of constraints was proposed by [7] in the context of the Electre Tri method. However, the resulting mathematical program is very complex (nonlinear and nonconvex constraints). A solution to circumvent this difficulty is to formulate partial inference programs, where only a subset of the parameters are considered as variables, while the remaining ones are fixed. In partial inference problems, if no combination of values for the inferred parameters is able to restore the statements contained in $S^{+}$and $S^{-}$, then the DM should wonder why. Then, he/she may either revise his/her statements or turn his/her attention to a different subset of parameters whose value may be inadequate. 
Among partial inference problems, previous research concerning Electre methods has focused mainly on inferring the weights and the cutting level. The problems involving the relation $S(a, b)$ can be solved using linear programs (LPs), but only if discordance is ignored, i.e., no veto phenomena occur and $N D(a, b)=1$ (e.g., see [8], [2] in the context of Electre Tri). However, when considering $S^{\prime}(a, b)$ or $S^{\prime \prime}(a, b)$, the weights and the cutting level can be inferred using LP, even in the presence of discordance (see [6]).

In the context of Electre Tri, a procedure exists to infer category limits, i.e., frontiers between categories and attached indifference and preference thresholds (everything else being fixed), assuming that no veto phenomena occur (see [9]). This procedure involves linear programs with $0-1$ variables.

We next present an approach to infer veto thresholds, when all other parameters have been fixed. For a more compact notation, we will write:

$$
\Delta_{j}(b, a)=g_{j}(b)-g_{j}(a)
$$

which is a constant value for each pair $(a, b) \in A^{2}$.

\section{Inference of veto-related parameters for a single criterion}

In this section, we consider that all the parameters are fixed, except the veto threshold of one criterion (let $i$ be its index). Indeed, contrarily to the weights, the veto thresholds are not interdependent, i.e., fixing the veto threshold for one criterion is not influenced by the value of the veto thresholds for the remaining criteria. Hence, the DM may wish to focus on the veto power of criteria, one criterion at a time. Being $v_{i}$ the only variable, all the requests from the DM can be satisfied iff the system (15) has a solution:

$$
\left\{\begin{aligned}
S(a, b) & \geq \lambda, \quad \forall(a, b) \in S^{+} \\
S(a, b) & <\lambda, \quad \forall(a, b) \in S^{-} \\
v_{i} & >p_{i}
\end{aligned}\right.
$$

\subsection{Inference of $v_{i}$ considering $S(a, b)$}

From (5) and (10), when only $v_{i}$ is considered as variable, $S(a, b)$ is equal to $N D_{i}(a, b)$ (which is a function of $v_{i}$ ) multiplied by a constant value $K_{i}(a, b)=C(a, b) . \prod_{j \in F \backslash\{i\}} N D_{j}(a, b)$. If this constant is lower than $\lambda$, then $a$ will not outrank $b$, even if there is no discordance on the $i$-th criterion, since $S(a, b) \leq K_{i}(a, b)$. Let us define a relation $a S_{-i} b$, meaning " $a S b$ when there is no discordance on the $i$-th criterion", or " $a S b$ is possible for some values of $v_{i}$ ":

$$
\begin{aligned}
a S_{-i} b & \Leftrightarrow\left(N D_{i}(a, b)=1 \Longrightarrow a S b\right) \\
& \Leftrightarrow K_{i}(a, b) \geq \lambda
\end{aligned}
$$

Since $K_{i}(a, b), C(a, b)$, and $\lambda$ are fixed constants, it is easy to perform the following preprocessing:

- if $\exists(a, b) \in S^{+}: \neg a S_{-i} b$ or $\exists(a, b) \in S^{-}: C(a, b)=1$ (which implies $S(a, b)=1$ ), then the system (15)has no solution. 
- all constraints associated with pairs $(a, b) \in S^{+}: C(a, b)=1$ or pairs $(a, b) \in S^{-}: \neg a S_{-i} b$ are redundant, because they will be respected for any value of $v_{i}$.

We now assume that this preprocessing has been performed. If the system was not found to be impossible and if the redundant constraints have been removed, then from (5), (6), and (10), the system (15)may be replaced by the following one (note that $K_{i}(a, b) \neq 0$ and $C(a, b)<1$ for all the pairs considered):

$$
\left\{\begin{aligned}
N D_{i}(a, b) & =\operatorname{Min}\left\{1, \frac{1-d_{i}(a, b)}{1-C(a, b)}\right\} \geq \frac{\lambda}{K_{i}(a, b)}, \quad \forall(a, b) \in S^{+}: a S_{-i} b \\
N D_{i}(a, b) & =\operatorname{Min}\left\{1, \frac{1-d_{i}(a, b)}{1-C(a, b)}\right\}<\frac{\lambda}{K_{i}(a, b)}, \quad \forall(a, b) \in S_{i}^{-}: a S_{-i} b \\
v_{i} & >p_{i}
\end{aligned}\right.
$$

Noting that $a S_{-i} b \Longrightarrow \lambda / K_{i}(a, b) \leq 1$, this system is equivalent to the following one:

$$
\left\{\begin{array}{rlrl}
1-d_{i}(a, b) & \geq(1-C(a, b)) \cdot \frac{\lambda}{K_{i}(a, b)}, & \forall(a, b) \in S^{+}: a S_{-i} b \\
1-d_{i}(a, b) & <(1-C(a, b)) \cdot \frac{\lambda}{K_{i}(a, b)}, & & \forall(a, b) \in S^{-}: a S_{-i} b \\
v_{i} & >p_{i}
\end{array}\right.
$$

If we now define $B_{i}(a, b)=1-\frac{(1-C(a, b)) \cdot \lambda}{K_{i}(a, b)}$, then the same system may be written as:

$$
\left\{\begin{aligned}
d_{i}(a, b) & \leq B_{i}(a, b), \quad \forall(a, b) \in S^{+}: a S_{-i} b \\
d_{i}(a, b) & >B_{i}(a, b), \quad \forall(a, b) \in S^{-}: a S_{-i} b \\
v_{i} & >p_{i}
\end{aligned}\right.
$$

where each $d_{i}(a, b)$ is a function of $v_{i}$ (recall that all other parameters are fixed) that yields a value in the interval $[0,1]$ (see (3) and Figure 1 ).

Since $\left.\left.\lambda / K_{i}(a, b) \in\right] 0,1\right]$ and $1>C(a, b) \geq \lambda \geq 0.5$, we conclude that $1>B_{i}(a, b)>0$. From (3), each constraint derived from a pair $(a, b) \in S^{+}: a S_{-i} b$ can be translated into a lower bound for $v_{i}$, and each constraint derived from a pair $(a, b) \in S^{-}: a S_{-i} b$ can be translated into an upper bound for $v_{i}$. Therefore, we may search for a solution to the system (19), knowing that $\left.d_{i}(a, b) \in\right] 0,1\left[\right.$ iff $d_{i}(a, b)=\frac{\Delta_{i}(b, a)-p_{i}}{v_{i}-p_{i}}:$

$$
\left\{\begin{array}{lll}
v_{i} \geq p_{i}+\frac{\Delta_{i}(b, a)-p_{i}}{B_{i}(a, b)}, & \forall(a, b) \in S^{+}: a S_{-i} b \\
v_{i}<p_{i}+\frac{\Delta_{i}(b, a)-p_{i}}{B_{i}(a, b)}, & \forall(a, b) \in S^{-}: a S_{-i} b \\
v_{i}>p_{i} &
\end{array}\right.
$$

Let $L_{i}$ denote the greatest of the lower bounds derived from $S^{+}$, and let $U_{i}$ denote the lowest of the upper bounds derived from $S^{-}$. Then the system (19) has no solution if $U_{i} \leq \max \left\{L_{i}, p_{i}\right\}$. Otherwise any value for $v_{i}$ in $\left[\max \left\{L_{i}, p_{i}\right\}, U_{i}\right]$ is acceptable, namely $v_{i}=\frac{U_{i}+\max \left\{L_{i}, p_{i}\right\}}{2}$.

\subsection{Inference of $u_{i}$ and $v_{i}$ considering $S^{\prime}(a, b)$}

In the specific case of the outranking relation $S^{\prime}(a, b)$ (see (11)), a new veto-related parameter $u_{i}$ has been introduced (see (8) and Figure 2). In this subsection we address the problem of inferring $u_{i}$ and $v_{i}$ simultaneously $\left(v_{i}>u_{i} \geq p_{i}\right)$. Note that if we considered that $u_{i}$ is fixed 
and $v_{i}$ is the only variable, then the process would be quite similar to the one followed in $\S 3.1$. Hence, the notations hereafter are similar:

- $K_{i}^{\prime}(a, b)=C(a, b) . \prod_{j \in F \backslash\{i\}} N D_{j}^{\prime}(a, b)$ (the product of the factors that do not depend on $u_{i}$ and $v_{i}$; this allows to write $\left.S^{\prime}(a, b)=N D_{i}^{\prime}(a, b) . K_{i}^{\prime}(a, b)\right)$;

- $a S_{-i}^{\prime} b \Leftrightarrow K_{i}^{\prime}(a, b) \geq \lambda\left(a S_{-i}^{\prime} b\right.$ iff $a S^{\prime} b$ is possible for some values of $u_{i}$ and $\left.v_{i}\right)$;

$-B_{i}^{\prime}(a, b)=1-\lambda / K_{i}^{\prime}(a, b)$

Following the reasoning of $\S 3.1$, we may perform a similar preprocessing that may detect that the problem is infeasible (if $\exists(a, b) \in S^{+}: \neg a S_{-i}^{\prime} b$ or $\exists(a, b) \in S^{-}: C(a, b)=1$ ) and allows to remove redundant constraints (those associated with pairs $(a, b) \in S^{+}: C(a, b)=1$ or pairs $\left.(a, b) \in S^{-}: \neg a S_{-i} b\right)$. After that, inferring $u_{i}$ and $v_{i}$ amounts at solving the following system of inequalities, where the variables $v_{i}$ and $u_{i}$ affect $d_{i}^{\prime}(a, b)$, whereas $B_{i}^{\prime}(a, b)$ are constants:

$$
\left\{\begin{aligned}
d_{i}^{\prime}(a, b) \leq B_{i}^{\prime}(a, b), \quad \forall(a, b) \in S^{+}: a S_{-i}^{\prime} b \\
d_{i}^{\prime}(a, b)>B_{i}^{\prime}(a, b), \quad \forall(a, b) \in S^{-}: a S_{-i}^{\prime} b \\
v_{i}>u_{i} \geq p_{i}
\end{aligned}\right.
$$

Since $\left.\left.\lambda / K_{i}^{\prime}(a, b) \in\right] 0,1\right]$, we conclude that $1>B_{i}(a, b) \geq 0$. Let us define the auxiliary notation:

$$
\begin{aligned}
& -S_{(B>0) i}^{+}=\left\{(a, b) \in S^{+}: a S_{-i}^{\prime} b \wedge 1>B_{i}^{\prime}(a, b)>0\right\} \\
& -S_{(B=0) i}^{+}=\left\{(a, b) \in S^{+}: a S_{-i}^{\prime} b \wedge B_{i}^{\prime}(a, b)=0\right\} .
\end{aligned}
$$

For each $(a, b) \in S_{(B=0) i}^{+}$we have a constraint $d_{i}^{\prime}(a, b)=0$ (note that $d_{i}^{\prime}(a, b)$ cannot be negative), which from (8) is equivalent to $\Delta_{i}(b, a) \leq u_{i}$. The remaining pairs $(a, b) \in S_{(B>0) i}^{+}$ and $(a, b) \in S_{i}^{-}: a S_{-i}^{\prime} b$ constrain $d_{i}^{\prime}(a, b)$ to be lower or higher (respectively) than a value in the interval $] 0,1\left[\right.$. Since $\left.d_{i}^{\prime}(a, b) \in\right] 0,1\left[\right.$ iff $d_{i}^{\prime}(a, b)=\frac{\Delta_{i}(b, a)-u_{i}}{v_{i}-u_{i}}$, the following system is equivalent to the previous one:

$$
\left\{\begin{aligned}
u_{i} \geq \Delta_{i}(b, a), \quad \forall(a, b) \in S_{(B=0) i}^{+} \\
v_{i} \geq u_{i}+\frac{\Delta_{i}(b, a)-u_{i}}{B_{i}^{\prime}(a, b)}, \quad \forall(a, b) \in S_{(B>0) i}^{+} \\
v_{i}<u_{i}+\frac{\Delta_{i}(b, a)-u_{i}}{B_{i}^{\prime}(a, b)}, \quad \forall(a, b) \in S^{-}: a S_{-i}^{\prime} b \\
v_{i}>u_{i} \geq p_{i}
\end{aligned}\right.
$$

In this case we may solve the following LP, where $\varepsilon$ is an arbitrary near-zero constant (to account for the strict inequalities) and the variables are $v_{i}, u_{i}$ and $\sigma$ :

$$
\begin{array}{ll}
\operatorname{Max} \sigma & \text { s.t. } \\
& \left\{\begin{array}{l}
u_{i} \geq \Delta_{i}(b, a)+\sigma, \quad \forall(a, b) \in S_{(B=0) i}^{+} \\
v_{i}+u_{i}\left(\frac{1}{B_{i}^{\prime}(a, b)}-1\right) \geq \frac{\Delta_{i}(b, a)}{U_{i}^{\prime}(a, b)}+\sigma, \forall(a, b) \in S_{(B>0) i}^{+} \\
v_{i}+u_{i}\left(\frac{1}{B_{i}^{\prime}(a, b)}-1\right) \leq \frac{\Delta_{i}(b, a)}{L_{i}^{\prime}(a, b)}-\sigma-\varepsilon, \forall(a, b) \in S^{-}: a S_{-i}^{\prime} b \\
v_{i}-\varepsilon \geq u_{i} \geq p_{i},
\end{array}\right.
\end{array}
$$

If the optimum value of the LP (22)is positive or null, then the system (20)has a solution, i.e., the optimum solution yields a value for $v_{i}$ and $u_{i}$ that respect all the statements provided by the DM. Otherwise, the system (20)has no solution. 


\subsection{Inference of $u_{i}$ and $v_{i}$ considering $S^{\prime \prime}(a, b)$}

Finally, we consider the outranking relation $S^{\prime \prime}(a, b)$. Let us define $M_{i}(a, b)=\min _{j \in F \backslash\{i\}} N D_{j}^{\prime}(a, b)$. As only $v_{i}$ is considered as variable, $M_{i}(a, b)$ is a constant value such that:

$$
S^{\prime \prime}(a, b)=C(a, b) \cdot \min \left\{M_{i}(a, b), 1-d_{i}^{\prime}(a, b)\right\} \leq C(a, b) \cdot M_{i}(a, b) .
$$

Following the reasoning of $\S 3.1$, we may define a relation analogous to $S_{-i}$ and $S_{-i}^{\prime}$ :

$$
a S_{-i}^{\prime \prime} b \Leftrightarrow C(a, b) M_{i}(a, b) \geq \lambda\left(a S_{-i}^{\prime \prime} b \text { iff } a S^{\prime \prime} b \text { is possible for some values of } u_{i} \text { and } v_{i}\right)
$$

We perform a similar preprocessing that may detect that the problem is infeasible (if $\exists(a, b) \in$ $S^{+}: \neg a S_{-i}^{\prime \prime} b$ or $\exists(a, b) \in S^{-}: C(a, b)=1$ ) and allows to remove redundant constraints (those associated with pairs $(a, b) \in S^{+}: C(a, b)=1$ or pairs $\left.(a, b) \in S^{-}: \neg a S_{-i}^{\prime \prime} b\right)$. Hence, we consider the system:

$$
\left\{\begin{aligned}
S^{\prime \prime}(a, b) & \geq \lambda, \quad \forall(a, b) \in S^{+}: a S_{-i}^{\prime \prime} b \\
S^{\prime \prime}(a, b) & <\lambda, \quad \forall(a, b) \in S^{-}: a S_{-i}^{\prime \prime} b \\
v_{i} & >u_{i} \geq p_{i}
\end{aligned}\right.
$$

From (23), each constraint $S^{\prime \prime}(a, b) \geq \lambda$ holds iff $M_{i}(a, b) \geq \lambda / C(a, b)$ and $1-d_{i}^{\prime}(a, b) \geq$ $\lambda / C(a, b)$. However, $a S_{-i}^{\prime \prime} b \Longrightarrow M_{i}(a, b) \geq \lambda / C(a, b)$; hence, $S^{\prime \prime}(a, b) \geq \lambda \Leftrightarrow 1-d_{i}^{\prime}(a, b) \geq$ $\lambda / C(a, b), \forall(a, b): a S_{-i}^{\prime \prime} b$. Therefore, if we now define $B_{i}^{\prime \prime}(a, b)=1-\lambda / C(a, b)$, then the system (26) can be written as:

$$
\left\{\begin{aligned}
& d_{i}^{\prime}(a, b) \leq B_{i}^{\prime \prime}(a, b), \forall(a, b) \in S^{+}: a S_{-i}^{\prime \prime} b \\
& d_{i}^{\prime}(a, b)>B_{i}^{\prime \prime}(a, b), \quad \forall(a, b) \in S^{-}: a S_{-i}^{\prime \prime} b \\
& v_{i}>u_{i} \geq p_{i}
\end{aligned}\right.
$$

This system is similar to the system (20) that we found in $\S 3.2$, hence we may proceed as proposed in that section: the process is the same, with a different definition for the relation $S_{-i}^{\prime}$, and the constants $B_{i}^{\prime}(a, b)$.

\section{Inference of all veto-related parameters simultaneously}

In this section we consider that all the parameters are fixed, except some of the veto thresholds, possibly all of them. This situation will occur when the DM does not wish to focus on the veto power of one criterion at a time. Let $V \subseteq F$ be the set of indices of the criteria whose veto threshold is not fixed. The criteria whose indices are in $F \backslash V$ are either fixed or do not possess any veto power $\left(v_{j}=\infty\right)$.

Being $v_{j}, j \in V$ the only variables, all the requests from the DM can be satisfied iff the system (27) has a solution:

$$
\left\{\begin{aligned}
S(a, b) & \geq \lambda, \quad \forall(a, b) \in S^{+} \\
S(a, b) & <\lambda, \quad \forall(a, b) \in S^{-} \\
v_{j} & >p_{j}, \quad \forall j \in V
\end{aligned}\right.
$$




\subsection{Inference of $v_{i}$ considering $S(a, b)$ or $S^{\prime}(a, b)$}

Let us consider the outranking $S(a, b)$ (see (10)) and define $K_{V}(a, b)=C(a, b) \cdot \prod_{j \in F \backslash V} N D_{j}(a, b)$, which is a constant value for $(a, b)$. We can now write $S(a, b)=K_{V}(a, b) \cdot \prod_{j \in V} N D_{j}(a, b) \leq$ $K_{V}(a, b)$. Let us define a relation $a S_{-V} b$, meaning " $a S b$ is possible for some values of $v_{j}, j \in V$ ":

$$
a S_{-V} b \Leftrightarrow K_{V}(a, b) \geq \lambda
$$

Since $K_{V}(a, b), C(a, b)$, and $\lambda$ are fixed constants, it is easy to perform the following preprocessing:

- if $\exists(a, b) \in S^{+}: \neg a S_{-V} b$ or $\exists(a, b) \in S^{-}: C(a, b)=1$ (which implies $S(a, b)=1$ ), then the system (27) has no solution.

- all constraints associated with pairs $(a, b) \in S^{+}: C(a, b)=1$ or pairs $(a, b) \in S^{-}: \neg a S_{-V} b$ are redundant, because they will be respected for any value of $v_{j}, j \in V$.

We now assume that this preprocessing has been performed. If the system was not found to be impossible and if the redundant constraints have been removed, then the system $(27)$ may be replaced by the following one, with $B_{V}(a, b)=\lambda / K_{V}(a, b)$ (note that $K_{V}(a, b) \neq 0$ and $C(a, b)<1$ for all the pairs considered):

$$
\left\{\begin{aligned}
\prod_{j \in V} N D_{j}(a, b) & \geq B_{V}(a, b), \quad \forall(a, b) \in S^{+}: a S_{-V} b \\
\prod_{j \in V} N D_{j}(a, b) & <B_{V}(a, b), \quad \forall(a, b) \in S^{-}: a S_{-V} b \\
v_{j} & >p_{j}, \quad \forall j \in V
\end{aligned}\right.
$$

This is a nonlinear system of inequalities, where the variables are $v_{j}, j \in V$, as arguments of $N D_{j}(a, b)(\operatorname{see} \S 1.3)$ :

$$
N D_{j}(a, b)=\min \left\{1, \frac{1-d_{j}(a, b)}{1-C(a, b)}\right\}=\min \left\{1, \frac{\min \left\{1, \max \left\{0, \frac{v_{j}-\Delta_{j}(b, a)}{v_{j}-p_{j}}\right\}\right\}}{1-C(a, b)}\right\}
$$

If $\Delta_{j}(b, a) \leq p_{j}$, then $N D_{j}(a, b)=1$, regardless of the value of $v_{j}$. Hence, if we denote $V_{a b}=\left\{j \in V: \Delta_{j}(b, a)>p_{j}\right\}$, we can write the system (29) above as:

$$
\left\{\begin{aligned}
\prod_{j \in V_{a b}} N D_{j}(a, b) & \geq B_{V}(a, b), \quad \forall(a, b) \in S^{+}: a S_{-V} b \\
\prod_{j \in V_{a b}} N D_{j}(a, b) & <B_{V}(a, b), \quad \forall(a, b) \in S^{-}: a S_{-V} b \\
v_{j} & >p_{j}, \quad \forall j \in V
\end{aligned}\right.
$$

Note also that:

$$
N D_{j}(a, b)=\min \left\{1, \frac{\max \left\{0, \frac{v_{j}-\Delta_{j}(b, a)}{v_{j}-p_{j}}\right\}}{1-C(a, b)}\right\}, \forall j \in V_{a b}
$$

We will now transform this system using logarithms. Let us define:

$$
f_{j}\left(a, b, v_{j}\right)=\log \max \left\{0.1, N D_{j}(a, b)\right\}, \forall j \in V_{a b}
$$


This definition considers that $N D_{j}(a, b)=0.1$, whenever its real value is below 0.1 (we need this to ensure we are taking the logarithm of a positive quantity). However, note that if $N D_{j}(a, b)<0.5$, for some $j \in V_{a b}$, then $S(a, b)<\lambda$, regardless of any other parameters, since $\lambda \geq 0.5$. Hence, this modification will not influence the results (any value in ]0,0.5[ could replace 0.1 ).

Now, the system (29) has a solution iff the following mathematical program has a nonnegative optimal value ( $\varepsilon$ is an arbitrary near-zero constant to account for the strict inequalities):

$\operatorname{Max} \sigma$ s.t.

$$
\left\{\begin{array}{l}
\sum_{j \in V_{a b}} f_{j}\left(a, b, v_{j}\right) \geq \log B_{V}(a, b)+\sigma, \quad \forall(a, b) \in S^{+}: a S_{-V} b \\
\sum_{j \in V_{a b}} f_{j}\left(a, b, v_{j}\right) \leq \log B_{V}(a, b)-\sigma-\varepsilon, \quad \forall(a, b) \in S^{-}: a S_{-V} b \\
v_{j} \geq p_{j}+\varepsilon, \quad \forall j \in V
\end{array}\right.
$$

The advantage of using logarithms is that we obtain a separable nonlinear program, which may be solved by 0-1 linear programming techniques. In the separable program (34), each function $f_{j}\left(a, b, v_{j}\right)$ may be approximated by a piecewise linear function of $v_{j}$. Since the feasible region is not convex, these problems may be solved either introducing some integer (0-1) variables or using a special branch and bound technique for dealing with SOS2 (special ordered sets of variables where at most two consecutive ones are non-zero) are used. [13] (chapter 7 and 9) and [14] (chapter 5 and 7) overview separable programs, including how to formulate them and solve them using either integer 0-1 programming or SOS2 branch and bound.

If we considered the outranking relation $S^{\prime}(a, b)$ (see (11)) instead of $S(a, b)$, the process would be analogous to the one described here, with the difference of placing $N D_{j}^{\prime}(a, b)$ instead of $N D_{j}(a, b)$. The only significant consequence is that $N D_{j}^{\prime}(a, b)$ depends also on the variables $u_{j}$ $(j \in V)$ (besides $\left.v_{j}\right)$, which increases the number of binary variables in the $0-1$ linear programs to solve.

\subsection{Inference of $u_{i}$ and $v_{i}$ considering $S^{\prime \prime}(a, b)$}

Considering the outranking relation $S^{\prime \prime}(a, b)$ (see (12)) and the variables $u_{j}$ and $v_{j}(j \in V)$, we will follow the similar reasoning, as we did for $S(a, b)$. The relation $a S_{-V}^{\prime \prime} b$ plays the same role as $a S_{-V} b$ in $\S 4.1$, but has a different definition:

$$
a S_{-V}^{\prime \prime} b \Leftrightarrow C(a, b) . \min _{j \in F \backslash V} N D_{j}^{\prime}(a, b) \geq \lambda
$$

We perform a similar preprocessing that may detect that the problem is infeasible (if $\exists(a, b) \in S^{+}: \neg a S_{-V}^{\prime \prime} b$ or $\left.\exists(a, b) \in S^{-}: C(a, b)=1\right)$ and allows to remove redundant constraints (those associated with pairs $(a, b) \in S^{+}: C(a, b)=1$ or pairs $\left.(a, b) \in S^{-}: \neg a S_{-V}^{\prime \prime} b\right)$. Hence, we consider the following system, with $B^{\prime \prime}(a, b)=\lambda / C(a, b)$ (recall the definition of $S^{\prime \prime}(a, b)$ (see (12)):

$$
\left\{\begin{aligned}
\min _{j \in F} N D_{j}^{\prime}(a, b) & \geq B^{\prime \prime}(a, b), \quad \forall(a, b) \in S^{+}: a S_{-V}^{\prime \prime} b \\
\min _{j \in F} N D_{j}^{\prime}(a, b) & <B^{\prime \prime}(a, b), \quad \forall(a, b) \in S^{-}: a S_{-V}^{\prime \prime} b \\
v_{j} & >u_{j} \geq p_{j}, \quad \forall j \in V
\end{aligned}\right.
$$

where the variables $u_{j}$ and $v_{j}(j \in V)$ are arguments of $N D_{j}^{\prime}(a, b)$. 
Noting that $N D_{j}^{\prime}(a, b)=\min \left\{1, \max \left\{0, \frac{v_{j}-\Delta_{j}(b, a)}{v_{j}-u_{j}}\right\}\right\}$ (see (7)-(8)), and since $a S_{-V}^{\prime \prime} b \Longrightarrow$ $\left.\left.B^{\prime \prime}(a, b) \in\right] 0,1\right]$, the system (36) is equivalent to the following one:

$$
\left\{\begin{aligned}
\min _{j \in F} \frac{v_{j}-\Delta_{j}(b, a)}{v_{j}-u_{j}} & \geq B^{\prime \prime}(a, b), \quad \forall(a, b) \in S^{+}: a S_{-V}^{\prime \prime} b \\
\min _{j \in F} \frac{v_{j}-\Delta_{j}(b, a)}{v_{j}-u_{j}} & <B^{\prime \prime}(a, b), \quad \forall(a, b) \in S^{-}: a S_{-V}^{\prime \prime} b \\
v_{j} & >u_{j} \geq p_{j}, \quad \forall j \in V
\end{aligned}\right.
$$

The values of $\frac{v_{j}-\Delta_{j}(b, a)}{v_{j}-u_{j}}$ are fixed for $j \in F \backslash V$ and variable for $j \in V$, hence we may readily verify that one of the fixed values makes a constraint (37.a) impossible to respect (hence there would be no solution) or makes a constraint (37.b) redundant (hence may be deleted).

We can now build a mathematical program to test if system (37) has a solution. Since $v_{j}>u_{j}$, each of the constraints (37.a)may be rewritten as:

$$
\begin{aligned}
& \min _{j \in F} \frac{v_{j}-\Delta_{j}(b, a)}{v_{j}-u_{j}}-B^{\prime \prime}(a, b) \geq 0 \\
\Leftrightarrow & \frac{v_{j}-\Delta_{j}(b, a)}{v_{j}-u_{j}}-B^{\prime \prime}(a, b) \geq 0, \forall j \in F \\
\Leftrightarrow & \left(1-B^{\prime \prime}(a, b)\right) \cdot v_{j}+B^{\prime \prime}(a, b) \cdot u_{j} \geq \Delta_{j}(b, a), \forall j \in F
\end{aligned}
$$

On the other hand, since $v_{j}>u_{j}$, each of the constraints (37.b) may be rewritten as:

$$
\begin{aligned}
& \min _{j \in F} \frac{v_{j}-\Delta_{j}(b, a)}{v_{j}-u_{j}}-B^{\prime \prime}(a, b)<0 \\
\Leftrightarrow & \exists j \in F: \frac{v_{j}-\Delta_{j}(b, a)}{v_{j}-u_{j}}-B^{\prime \prime}(a, b)<0 \\
\Leftrightarrow & \exists j \in F:\left(1-B^{\prime \prime}(a, b)\right) \cdot v_{j}+B^{\prime \prime}(a, b) \cdot u_{j}<\Delta_{j}(b, a) \\
\Leftrightarrow & \left\{\begin{array}{l}
\left(1-B^{\prime \prime}(a, b)\right) \cdot v_{j}+B^{\prime \prime}(a, b) \cdot u_{j}+M \cdot \delta_{j a b}<M+\Delta_{j}(b, a), \forall j \in F \\
\sum_{j \in F} \delta_{j a b} \geq 1 \\
\delta_{j a b} \in\{0,1\}, \forall j \in F
\end{array}\right.
\end{aligned}
$$

where $M$ is a large positive constant greater than $\frac{v_{j}-\Delta_{j}(b, a)}{v_{j}-u_{j}}, \forall j \in F, \forall(a, b) \in S^{-}: a S_{-V}^{\prime \prime} b$. The system (40) uses binary variables to account for the disjunctive nature of (39). Note that $\sum_{j \in F} \delta_{j a b} \geq 1$ forces at least one of the binary variables $\delta_{j a b}$ to be have the value 1 , thus forcing (39). Considering these transformations, the system (37) has a solution iff the following 0-1 linear program has a non-negative optimal value ( $\varepsilon$ is an arbitrary near-zero constant):

$\operatorname{Max} \sigma$ s.t.

$$
\begin{cases}\left(1-B^{\prime \prime}(a, b)\right) \cdot v_{j}+B^{\prime \prime}(a, b) \cdot u_{j} \geq \Delta_{j}(b, a) & +\sigma, \\ & \forall j \in F,(a, b) \in S^{+}: a S_{-V}^{\prime \prime} b \\ \left(1-B^{\prime \prime}(a, b)\right) \cdot v_{j}+B^{\prime \prime}(a, b) \cdot u_{j}+M . \delta_{j a b} \leq & M+\Delta_{j}(b, a)-\sigma-\varepsilon, \\ & \forall j \in F,(a, b) \in S^{-}: a S_{-V}^{\prime \prime} b \\ \sum_{j \in F} \delta_{j a b} \geq 1, \forall(a, b) \in S^{-}: a S_{-V}^{\prime \prime} b & \\ v_{j}-\varepsilon \geq u_{j} \geq p_{j}, \quad \forall j \in V & \\ \delta_{j a b} \in\{0,1\}, \forall j \in F,(a, b) \in S^{-}: a S_{-V}^{\prime \prime} b, & \sigma \text { free. }\end{cases}
$$




\section{$5 \quad$ Illustrative example}

In this section, we present an example that illustrates the procedures presented in $\S 3$ and $\S 4$. This example deals with a multiple criteria sorting problem using the Electre Tri method (see [12] and [15]). Within this framework, we infer the veto thresholds (the value of all other parameters being fixed) from assignment examples using Electre Tri pessimistic assignment rule. Let us note that this problem amounts at inferring a relation $S$ from outranking statements (see $\S 2$ ) as in it holds $a$ is assigned to $C_{k}$ iff $a S b_{k} \wedge \neg a S b_{k+1}$.

\subsection{Scheme of the experiment}

The experiment will proceed as follows. First, we define an Electre Tri model by specifying all preference parameters (including veto thresholds). Applying this model allows us to assign each alternative to a specific category. Next, we consider these results as assignment examples. These examples are used as an input of the inference procedures defined in $\S 3$ and $\S 4$ where we consider one/several veto-related parameters unknown. This experimental scheme (see Figure 3) enables us to compare the inferred model to the initial one, to analyze the behavior of the inference procedures.

Let us consider a sorting problem in which alternatives have to be assigned to three categories, good $\succ$ medium $\succ$ bad, defined by two profiles, $b_{1}$ and $b_{2}(B=\{1,2\})$, taking into account their evaluations on 7 criteria, $g_{1}, \ldots, g_{7}(F=\{1, \ldots, 7\})$. The evaluations on each criterion take their values in the interval [0,100]. The Electre Tri model is defined by the values for preference parameters specified in Table 1 . Note that the veto parameters are $v_{j}\left(b_{1}\right)$ and $v_{j}\left(b_{2}\right)$, i.e., defined as associated with the limits of the categories. Electre Tri pessimistic assignment rule (using these parameters and posing $\lambda=0.61$ ) defines the 6 assignment examples given in Table 2 .
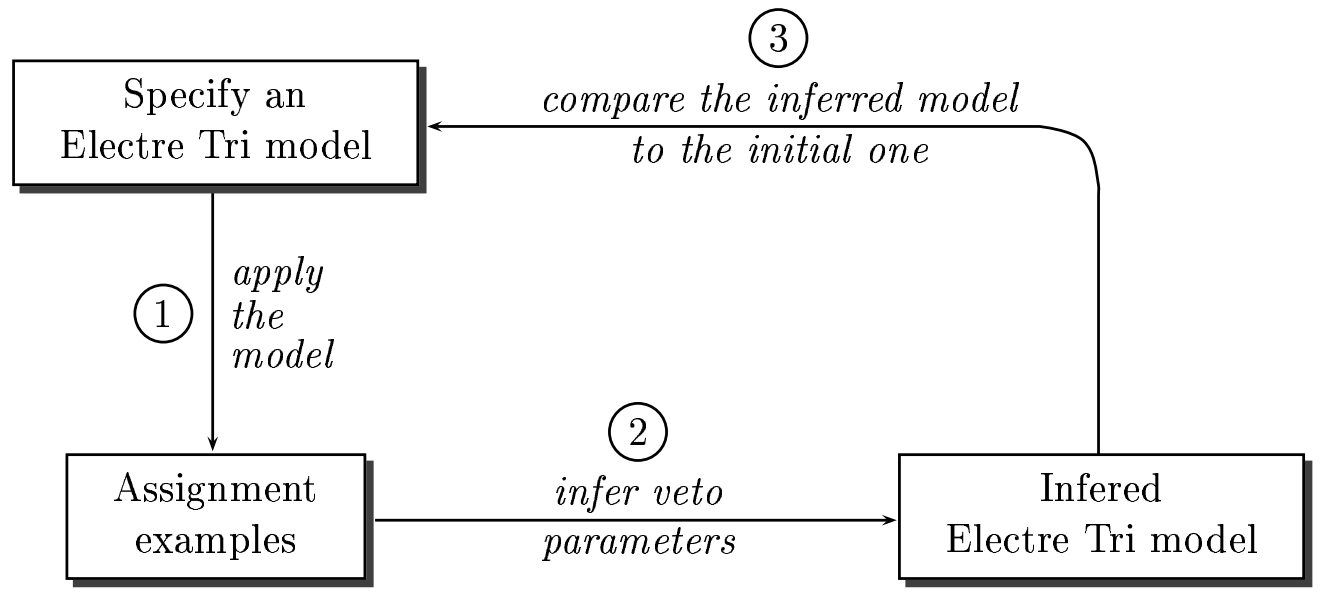

FIG. 3: Experimental scheme 


\begin{tabular}{|l|c|c|c|c|c|c|c|}
\cline { 2 - 8 } \multicolumn{1}{c|}{} & $g_{1}$ & $g_{2}$ & $g_{3}$ & $g_{4}$ & $g_{5}$ & $g_{6}$ & $g_{7}$ \\
\hline$w_{j}$ & 0.143 & 0.143 & 0.143 & 0.143 & 0.143 & 0.143 & 0.143 \\
\hline$g_{j}\left(b_{1}\right)$ & 33 & 33 & 33 & 33 & 33 & 33 & 33 \\
$q_{j}\left(b_{1}\right)$ & 3 & 3 & 3 & 3 & 3 & 3 & 3 \\
$p_{j}\left(b_{1}\right)$ & 5 & 5 & 5 & 5 & 5 & 5 & 5 \\
$v_{j}\left(b_{1}\right)$ & 33 & 33 & 33 & $\infty$ & $\infty$ & $\infty$ & $\infty$ \\
\hline$g_{j}\left(b_{2}\right)$ & 66 & 66 & 66 & 66 & 66 & 66 & 66 \\
$q_{j}\left(b_{2}\right)$ & 3 & 3 & 3 & 3 & 3 & 3 & 3 \\
$p_{j}\left(b_{2}\right)$ & 5 & 5 & 5 & 5 & 5 & 5 & 5 \\
$v_{j}\left(b_{2}\right)$ & 33 & 33 & 33 & $\infty$ & $\infty$ & $\infty$ & $\infty$ \\
\hline
\end{tabular}

TAB. 1: preference parameters

\begin{tabular}{|c|c|c|c|c|c|c|c|c|}
\cline { 2 - 9 } \multicolumn{1}{c|}{} & $g_{1}$ & $g_{2}$ & $g_{3}$ & $g_{4}$ & $g_{5}$ & $g_{6}$ & $g_{7}$ & Category \\
\hline$a_{1}$ & 39 & 55 & 59 & 65 & 65 & 65 & 65 & $C_{2}$ \\
$a_{2}$ & 45 & 55 & 59 & 65 & 65 & 65 & 65 & $C_{3}$ \\
$a_{3}$ & 49 & 55 & 59 & 65 & 65 & 65 & 65 & $C_{3}$ \\
$a_{4}$ & 7 & 27 & 29 & 25 & 65 & 65 & 65 & $C_{1}$ \\
$a_{5}$ & 6 & 27 & 29 & 65 & 65 & 65 & 65 & $C_{1}$ \\
$a_{6}$ & 8 & 6.5 & 29 & 65 & 65 & 65 & 65 & $C_{2}$ \\
\hline
\end{tabular}

TAB. 2: Set of assignment examples

These assignment examples result in the following positive and negative outranking statements: $S^{+}=\left\{\left(a_{1}, b_{1}\right),\left(a_{2}, b_{2}\right),\left(a_{3}, b_{2}\right),\left(a_{6}, b_{1}\right)\right\}$ and $S^{-}=\left\{\left(a_{1}, b_{2}\right),\left(a_{4}, b_{1}\right),\left(a_{5}, b_{1}\right),\left(a_{6}, b_{2}\right)\right\}$.

\section{2 inferring one veto using the relation $S$}

We first infer the veto parameters for criterion $g_{1}$, i.e., $v_{1}\left(b_{1}\right)$ and $v_{1}\left(b_{2}\right)$. Following the method defined in $\S 3.1$, the sets $S^{+}$and $S^{-}$lead to $v_{1}\left(b_{1}\right) \in[32.7,35.4]$ and $v_{1}\left(b_{2}\right) \in[30.2,39.7]$. These intervals result from the computations given in Table 3 and Table 4 . As expected, the values for $v_{1}\left(b_{1}\right)=33$ and $v_{1}\left(b_{2}\right)=33$ in the initial model (the one from which the assignment examples were derived) are contained in the computed intervals.

\begin{tabular}{|l|c|c|c|c|c|c|c|}
\hline \multicolumn{1}{|l|}{$S^{+}$} & \multicolumn{1}{l|}{} \\
\hline$a_{i}$ & $b_{h}$ & $g_{1}\left(a_{i}\right)$ & $g_{1}\left(b_{h}\right)$ & $K_{1}\left(a_{i}, b_{h}\right)$ & Inconsistent? & $B_{1}\left(a_{i}, b_{h}\right)$ & $v_{1}^{\text {min }}\left(b_{h}\right)$ \\
\hline$a_{1}$ & $b_{1}$ & 39 & 33 & 1.000 & no & 1.000 & -6.00 \\
$a_{2}$ & $b_{2}$ & 45 & 66 & 0.625 & no & 0.634 & 30.24 \\
$a_{3}$ & $b_{2}$ & 45 & 66 & 0.625 & no & 0.634 & 23.93 \\
$a_{6}$ & $b_{1}$ & 8 & 33 & 0.6875 & no & 0.723 & 32.67 \\
\hline
\end{tabular}

TAB. 3: Computation of lower bounds for $v_{1}\left(b_{h}\right)$ 


\begin{tabular}{|c|c|c|c|c|c|c|c|}
\hline \multicolumn{2}{|l|}{$S^{-}$} & \multicolumn{7}{|l|}{} \\
\hline$a_{i}$ & $b_{h}$ & $g_{1}\left(a_{i}\right)$ & $g_{1}\left(b_{h}\right)$ & $K_{1}\left(a_{i}, b_{h}\right)$ & Redundant? & $B_{1}\left(a_{i}, b_{h}\right)$ & $v_{1}^{\text {max }}\left(b_{h}\right)$ \\
\hline$a_{1}$ & $b_{2}$ & 39 & 66 & 0.625 & no & 0.634 & 39.70 \\
$a_{4}$ & $b_{1}$ & 7 & 33 & 0.5625 & yes & - & - \\
$a_{5}$ & $b_{1}$ & 6 & 33 & 0.6875 & no & 0.723 & 35.44 \\
$a_{6}$ & $b_{2}$ & 8 & 66 & 0 & yes & - & - \\
\hline
\end{tabular}

TAB. 4: Computation of upper bounds for $v_{1}\left(b_{h}\right)$

\section{3 inferring one veto using the relation $S^{\prime}$}

In the case of the outranking relation $S^{\prime}$ (see (11)), a new veto-related parameter $u_{i}$ is introduced (see (8) and Figure 2). If we pose $u_{i}=p_{j}+0.75\left(v_{j}-p_{j}\right)\left(v_{i}\right.$ being the only variable), then the calculations are very similar to the preceding case and lead to the following intervals: $v_{1}\left(b_{1}\right) \in[31.3,33.3]$ and $v_{1}\left(b_{2}\right) \in[26.2,34.1]$.

If we consider the threshold $u_{1}$ as a parameter to be inferred, and we impose both thresholds to be equal (i.e., $u_{1}\left(b_{1}\right)=u_{1}\left(b_{2}\right)=u_{1}$ and $\left.v_{1}\left(b_{1}\right)=v_{1}\left(b_{2}\right)=v_{1}\right)$, the mathematical program to be solved is the following:

$$
\begin{aligned}
& \operatorname{Max} \sigma \text { s.t. } \\
& \qquad \begin{array}{l}
1.56 u_{1}+v_{1}-\sigma \geq-15.38 \\
40.66 u_{1}+v_{1}-\sigma \geq 875 \\
40.66 u_{1}+v_{1}-\sigma \geq 708.33 \\
7.87 u_{1}+v_{1}-\sigma \geq 221.77 \\
-40.39 u_{1}-v_{1}-\sigma \geq-1117.55+\varepsilon \\
-7.86 u_{1}-v_{1}-\sigma \geq-239.21+\varepsilon \\
u_{j} \geq p_{j}\left(g_{j}\right)+\varepsilon \\
v_{j} \geq u_{j}
\end{array}
\end{aligned}
$$

In this mathematical program, the four first constraints result from the positive outranking statements in $S^{+}\left(a_{1} S b_{1}, a_{2} S b_{2}, a_{3} S b_{2}, a_{6} S b_{1}\right)$ and the two following ones result from negative outranking in $S^{-}\left(\neg a_{4} S b_{1}\right.$ and $\neg a_{6} S b_{2}$, the two other negative outranking statements being redundant). At the optimum $v_{1}=25.6$ and $u_{1}=25.5$.

\section{4 inferring one veto using the relation $S^{\prime \prime}$}

To illustrate the case of the outranking relation $S^{\prime \prime}$ we pose $u_{i}=p_{j}+0.75\left(v_{j}-p_{j}\right)\left(v_{i}\right.$ being the only variable). In this case, the calculations are similar to $\$ 5.2$ and lead to the following intervals: $v_{1}\left(b_{1}\right) \in[30.7,33.3]$ and $v_{1}\left(b_{2}\right) \in[26.2,34.1]$.

\section{5 inferring several veto using the relation $S^{\prime \prime}$}

Let us suppose that the DM wants to infer all veto simultaneously. In this case, we will suppose that $u_{j}=p_{j}+0.75\left(v_{j}-p_{j}\right)$ ( $v_{j}$ being the only variables). As the DM considers that only the first three criteria can have a veto effect, we infer $v_{1}, v_{2}$ and $v_{3}$ from the assignment 
examples. In this case, the mathematical program to be solved is given below. At the optimum, it holds: $v_{1}\left(b_{1}\right)=32.0, v_{1}\left(b_{2}\right)=27.5, v_{2}\left(b_{1}\right)=33.9, v_{2}\left(b_{2}\right)=14.3, v_{3}\left(b_{1}\right)=5.0$ and $v_{3}\left(b_{2}\right)=22.2$.

$\operatorname{Max} \sigma$ s.t.

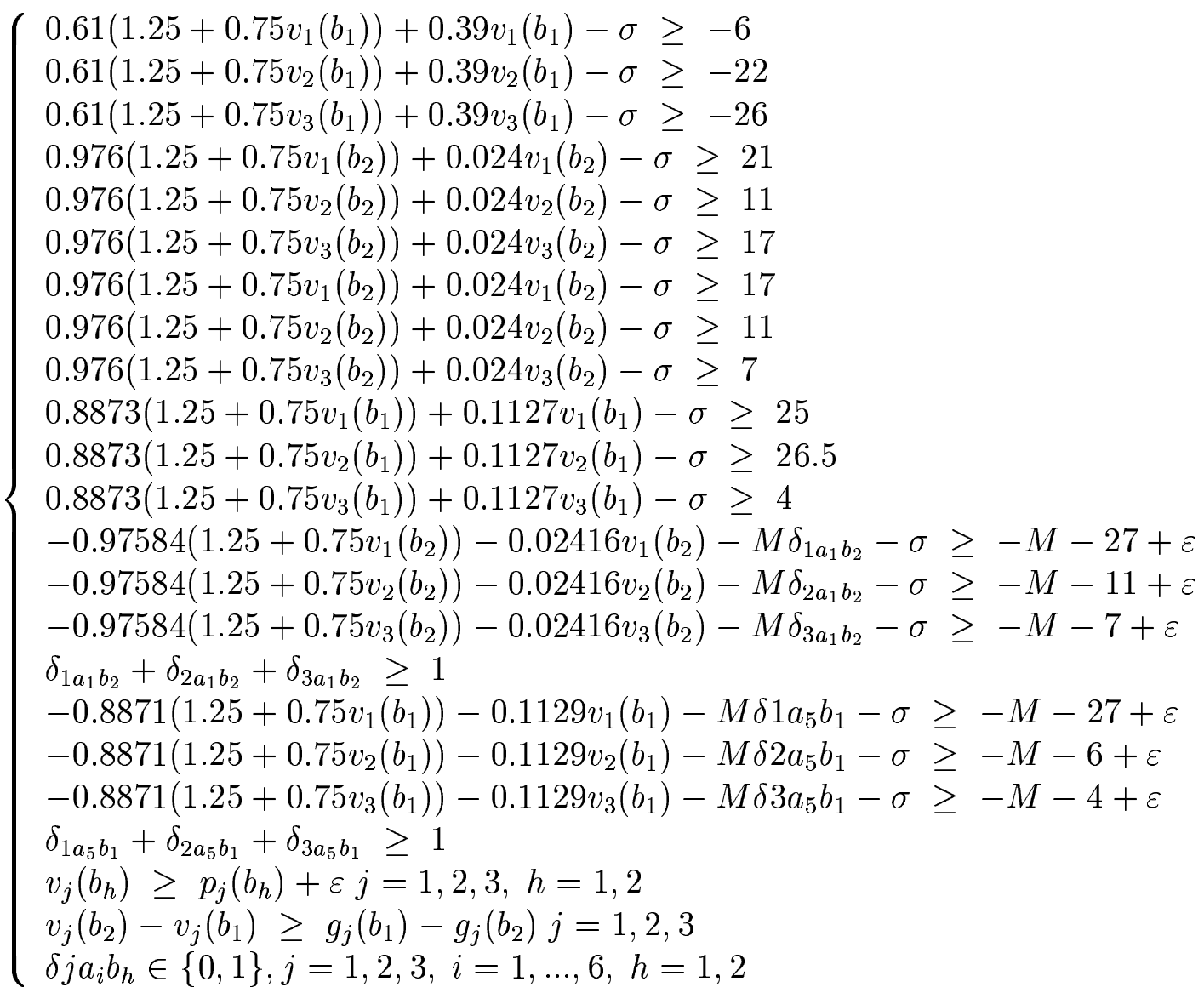

\section{Conclusion}

This paper presents a contribution to a methodology to infer the parameter values of an Electre model from crisp outranking statements provided by the DM (statements that the model should restore). This is a difficult problem when all parameters have to be inferred simultaneously, hence we limit ourselves to an interactive process of partial inference problems. Partial inference problems are frequently a wise choice as regards the interaction with a DM, since they allow greater control and comprehension of the interactive process.

This paper focusses on the inference of the discordance-related parameters (veto thresholds), thus complementing previous work on the inference of the concordance-related parameters (weights, cutting level and limits of categories). In [6] we used two variants of the original valued outranking relation $S$ (denoted $S^{\prime}$ and $S^{\prime \prime}$ ) to simplify inference problems. In this paper, we show that regarding the inference of the veto thresholds, $S$ and $S^{\prime}$ originate mathematical programs of similar complexity, while $S^{\prime \prime}$ yields simpler versions. Table 5 summarizes the type of mathematical programs corresponding to each situation. Although we have considered veto thresholds as independent from the performances, this table applies to the more general case 
where thresholds are affine functions of $g_{j}($.$) (see details in [1]).$

\begin{tabular}{|l|l|l|l|}
\cline { 2 - 4 } \multicolumn{1}{c|}{} & $S$ & $S^{\prime}$ & $S^{\prime \prime}$ \\
\hline $\begin{array}{l}\text { Inference of weights } \\
\text { and cutting level }\end{array}$ & $\begin{array}{l}\text { Global (non-convex) } \\
\text { programming }\end{array}$ & $\begin{array}{l}\text { Linear program- } \\
\text { ming }\end{array}$ & $\begin{array}{l}\text { Linear program- } \\
\text { ming }\end{array}$ \\
\hline $\begin{array}{l}\text { Inference of veto for } \\
\text { a single criterion }\end{array}$ & Linear programming & $\begin{array}{l}\text { Linear program- } \\
\text { ming }\end{array}$ & $\begin{array}{l}\text { Linear program- } \\
\text { ming }\end{array}$ \\
\hline $\begin{array}{l}\text { Inference of veto for } \\
\text { all the criteria }\end{array}$ & $\begin{array}{l}\text { Separable nonlinear } \\
\text { programming }\end{array}$ & $\begin{array}{l}\text { Separable nonli- } \\
\text { near programming }\end{array}$ & $\begin{array}{l}\text { 0-1 linear pro- } \\
\text { gramming }\end{array}$ \\
\hline
\end{tabular}

TAB. 5: Mathematical programs corresponding to the different veto inference problems

Illustrative examples provided in $\S 5$ show how the inference procedures can be used in a sorting problem based on an Electre Tri model. Further research should be conducted in order to study empirically the behavior of the inference procedures.

Acknowledgements: This work has benefited from the grant $n^{\circ} 500 \mathrm{~B} 4$ (ICCTI/Ambassade de France au Portugal). The authors are grateful to Bernard Roy whose remarks greatly improved the quality of this paper.

\section{Références}

[1] L.C. Dias and V. Mousseau. Inferring ELECTRE's veto-related parameters from outranking examples. Research report n5/2002, INESC Coimbra, 2002.

[2] L.C. Dias, V. Mousseau, J. Figueira, and J. Clímaco. An aggregation/disaggregation approach to obtain robust conclusions with ELECTRE TRI. European Journal of Operational Research, 138(2):332-348, April 2002.

[3] E. Jacquet-Lagrèze and J. Siskos. Assessing a set of additive utility functions for multicriteria decision-making, the UTA method. European Journal of Operational Research, 10(2):151-164, 1982.

[4] E. Jacquet-Lagrèze and Y. Siskos. Preference disaggregation: 20 years of MCDA experience. European Journal of Operational Research, 130(2):233-245, April 2001.

[5] L.N. Kiss, J.-M. Martel, and R. Nadeau. ELECCALC - an interactive software for modelling the decision maker's preferences. Decision Support Systems, 12(4-5):757-777, November 1994. 
[6] V. Mousseau and L. Dias. Valued outranking relations in ELECTRE providing manageable disaggregation procedures. Cahier du LAMSADE $n^{\circ} 189$, Université de Paris-Dauphine, December 2001.

[7] V. Mousseau and R. Slowinski. Inferring an EleCtre TRI model from assignment examples. Journal of Global Optimization, 12(2):157-174, 1998.

[8] V. Mousseau, R. Slowinski, and P. Zielniewicz. A user-oriented implementation of the ELECTRE TRI method integrating preference elicitation support. Computers $\&$ Operations Research, 27(7-8):757-777, June 2000.

[9] A. Ngo The and V. Mousseau. Using assignment examples to infer category limits for the Electre Tri method. Cahier du LAMSADE no 168 , Université de Paris-Dauphine, 2000.

[10] B. Roy. ELECTRE III : un algorithme de classement fondé sur une représentation floue des préférences en présence de critères multiples. Cahiers du CERO, 20(1):3-24, 1978.

[11] B. Roy. The outranking approach and the foundations of ELECTRE methods. Theory and Decision, 31(1):49-73, 1991.

[12] B. Roy and D. Bouyssou. Aide multicritère à la décision: Méthodes et cas. Economica, Paris, 1993.

[13] H.P. Williams. Model building in mathematical programming. Wiley, Chichester, 1990. third edition.

[14] H.P. Williams. Model solving in mathematical programming. Wiley, Chichester, 1993.

[15] W. Yu. Aide multicritère à la décision dans le cadre de la problématique du tri: concepts, méthodes et applications. PhD thesis, Université Paris-Dauphine, 1992.

[16] C. Zopounidis and M. Doumpos. PREFDIS: a multicriteria decision support system for sorting decision problems. Computers \& Operations Research, 27(7-8):779-797, June 2000. 